\title{
Comments on Nomenclature of COVID-19
}

\author{
Li Liu ${ }^{1 *}$ and Yumin Huang-Link ${ }^{2}$ \\ Medical College of Wuhan University in Science and Technology, China \\ Division of Neurology and Biomedical and Clinical Sciences, Faculty of Medicine and Health Sciences, Linköping University, China
}

*Corresponding author: Li Liu, Medical College of Wuhan University of Science and Technology, 947 Heping Avenue, Qingshan District, Wuhan, 430081, China.

To Cite This Article: Li Liu, Comments on Nomenclature of COVID-19. 2020 - 9(6). AJBSR.MS.ID.001457. DOI: 10.34297/AJBSR.2020.09.001457.

Received: 眥August 05, 2020; Published: 㘹 August 14, 2020

\section{Introduction}

Scientists and medical doctors have been contributing largely to identify novel coronavirus 2 as the cause of the severe acute respiratory syndrome (SARS-COV-2) which emerged in large numbers of infected people in Wuhan City since Dec. 2019 [13]. Scientists quickly sequenced SARS-COV-2 and shared their knowledge with the world $[3,4]$. Searching for vaccine and specific therapy is the ongoing global public health concern to prevent future spreading [5]. The new research, clinical and epidemiological data on SARS-COV-2 are continuing to explode. We need to make consensus on nomenclature in SARS-COV-2 studies correctly and precisely.

Coronavirus (COVs) is one of major viruses that primarily targets the human respiratory system. Previous COV outbreaks were seen in SARS 2003 in Asia, and Middle East respiratory syndrome (MERS) 2012. Acute respiratory illness has been dominant in COVs caused diseases with fever, cough, sore throat, breathlessness, fatigue, malaise [4]. In the case of COV-2 the clinical feature is similar. Patients with clinical symptoms and SARS-COV-2 positive are diagnosed as coronaviruses disease 2019 (COVID-19). But with the increasing test of naso-pharyngeal swab we realize that people with SARS-COV-2 positive can be asymptomatic. This group may be defined as SARS-COV-2 carrier. On the other hand, people with SARS-COV-2 negative can have typical COVID-19 clinical manifestation, the chest X-ray showing bilateral infiltrates and laboratory features with increased levels of C-reactive protein (CRP), inflammatory cytokines like interleukin-6, ferritin, D-dimer, neutrophilia and lymphocytopenia. Such cases may be defined as suspected COVID-19. The COVD-19 can behavior with encephalitis, polycranial neuritis, Miller-Fisher syndrome, Guillain-Barré
Syndrome, cytokine release syndrome, endo-carditis or multiorgan etc $[5,6]$, these groups may be defined as SARS-COV-2 syndrome. If there is an evidence of lower respiratory tract involvement with abnormal chest X-ray or CT scans, the COVID-19 pneumonia or severe acute respiratory syndrome coronavirus 2 (SARS-CoV-2) should be applied to indicate severe consequence with possible respiratory failure [2-4].

In Europe or the United States, there is increased evidence that COVD-19 can behavior with olfactory reduction, diarrhea, encephalitis, endo-carditis or multiorgan failure with or without SARS-COV-2 symptoms [7]. In such cases differential diagnoses is urged clinically. These patients should be treated in isolation until COV-2 and other relevant laboratory tests have been performed and interpreted correctly before the patients are moved out from the COVID-19/Pandemic unit.

In summary, COVID-19 serves as general diagnosis to define COV-2 related symptoms including SARS-COV-2. SARS-COV-2 is specifically applied to indicate severe acute respiratory syndrome caused by COV-2. Those who have typical COVID-19 features without positive COV-2 may be defined as suspected COVID-19. Those who have no symptoms, but positive COV-2 may be defined as COV-2 carrier. Certainly, future antibody (IgG and IgM against COV-2) test will broaden diagnosis and prognosis of COVID-19.

\section{Acknowledgement}

We are thankful for revision and import from Prof. Hans Link, Dept of Neurosciences, Karolinska Institute, Stockholm, Sweden.

\section{Conflict of Interest Statement}

The authors have no competing interests to declare. No financial support 


\section{References}

1. Wu Z, McGoogan JM. (2020) Characteristics of and Important Lessons from the Coronavirus Disease 2019 (COVID-19) Outbreak in China: Summary of a Report of 72314 Cases from the Chinese Center for Disease Control and Prevention.

2. Huang C, Wang Y, Li X, Lili Ren, Jianping Zhao, et al. (2020) Clinical features of patients infected with 2019 novel coronavirus in Wuhan, China. Lancet. 395(10223): 497-506.

3. Zhai P, Ding Y, Wu X, Junke Long, Yanjun Zhong, et al. (2020) The epidemiology, diagnosis and treatment of COVID-19. Int J Antimicrob Agents 55(5): 105955

4. Chen H, Guo J, Wang C, Fan Luo, Xuechen Yu, et al. (2020) Clinical characteristics and intrauterine vertical transmission potential of COVID-19 infection in nine pregnant women: a retrospective review of medical records. Lancet 395(10226): 809-815.

5. Moore JB, June CH (2020) Cytokine release syndrome in severe COVID-19. Science 368(6490): 473-474.

6. Gutiérrez-Ortiz C, Méndez A, Rodrigo-Rey S, Eduardo San Pedro-Murillo, Laura Bermejo-Guerrero, et al. (2020) Miller Fisher Syndrome and polyneuritis cranialis in COVID-19. Neurology.

7. Helms J, Stéphane Kremer, Hamid Merdji, Raphaël Clere-Jehl, Malika Schenck, et al. (2020) Neurologic Features in Severe SARS-CoV-2 Infection. N Engl J Med 382(23): 2268-2270. 